\title{
Application de l'interaction acousto-optique dans les solides à l'analyse ultra- rapide d'un spectre lumineux
}

\author{
F. Treffé et C. Carles \\ Conservatoire National des Arts et Métiers, Laboratoire d'Acoustique et d'Eclairage-Optique-Image, \\ 292, rue Saint-Martin, 75003 Paris, France
}

(Reçu le 25 février 1980, révisé le 28 avril 1980, accepté le 6 mai 1980)

\begin{abstract}
Résumé. - Les caractéristiques intrinsèques et expérimentales d'un dispositif spectroscopique à balayage ultrarapide sont rappelées et calculées dans le cas de l'expérience décrite. Dispersion et balayage de la lumière sont assurés par un déviateur acousto-optique constitué d'un cristal de $\mathrm{PbMoO}_{4}$ et travaillant en ondes longitudinales. Les fréquences acoustiques varient de 150 à $300 \mathrm{MHz}$.

La source utilisée pour effectuer les mesures est un laser à argon ionisé. Le pouvoir séparateur du dispositif décrit dans cet article est en moyenne de 4,5 $\AA$ pour les raies les plus intenses de ce laser. La résolution est indépendante de la vitesse de balayage qui affecte néanmoins la largeur spectrale analysable. Le dispositif permet de balayer le spectre visible en un temps de $100 \mu \mathrm{s}$, mais avec un circuit de détection adapté, il est possible d'atteindre des temps inférieurs.
\end{abstract}

\begin{abstract}
Intrinsic and experimental features of a high speed spectroscopic device are defined and calculated. For the dispersion and scanning of light, we use $\mathrm{PbMoO}_{4}$ Bragg cell working with longitudinal acoustic waves. The acoustic frequencies vary in the 150 to $300 \mathrm{MHz}$ range.

The light source used in this experiment is an Ar ion laser. The device described in this paper gives a mean resolving power of $4.5 \AA$ for the main lines available from the laser. The resolution is not depending on the scanning rate which affects the analysing spectral range. With an appropriate detection set up, the whole visible spectrum range can be analysed in $100 \mu$ s or less.
\end{abstract}

1. Introduction. - Depuis les premières expériences de déviation de la lumière par interaction acousto-optique $[1,2,3]$, de nombreux appareils ont été réalisés. Tous ces appareils sont capables de dévier de la lumière monochromatique dans un nombre de directions plus ou moins important. A partir de ce principe, nous nous proposons de décrire un dispositif d'analyse spectrale d'une source cohérente par un balayage ultra-rapide et d'en déterminer les possibilités.

D'après la relation de Bragg [4] :

$$
\sin \theta=\frac{\lambda F}{V}
$$

il est intéressant de remarquer que l'angle $\theta$ de déviation du faisceau lumineux incident, dépend non seulement de la fréquence acoustique $F$, mais aussi de la longueur d'onde $\lambda$ de la lumière déviée $(V$ : vitesse de propagation des ultrasons dans le milieu d'inter- action). Par conséquent si la lumière incidente est polychromatique, le déviateur se comporte, pour une fréquence acoustique donnée, comme un spectromètre optique. Si la fréquence acoustique varie continûment dans un intervalle $\Delta F$, le faisceau dévié, pour une longueur d'onde lumineuse $\lambda$, balaye un angle $\Delta \theta$ du plan d'incidence.

Ainsi le réseau à pas variable que constitue le déviateur, assure à la fois la décomposition spectrale $\mathrm{du}$ faisceau lumineux et son balayage devant un dispositif d'analyse.

A partir de ce principe, Yano et Watanabe ont réalisé un filtre accordable utilisant un déviateur en paratellurite $\left(\mathrm{TeO}_{2}\right)$ travaillant en ondes acoustiques transverses $[5,6]$. Notre étude s'est orientée vers une application de spectrométrie ultra-rapide à partir d'un déviateur en $\mathrm{PbMoO}_{4}$ et avec une propagation longitudinale des ondes acoustiques. Pour cela nous avons utilisé le montage schématisé sur la figure 1. 


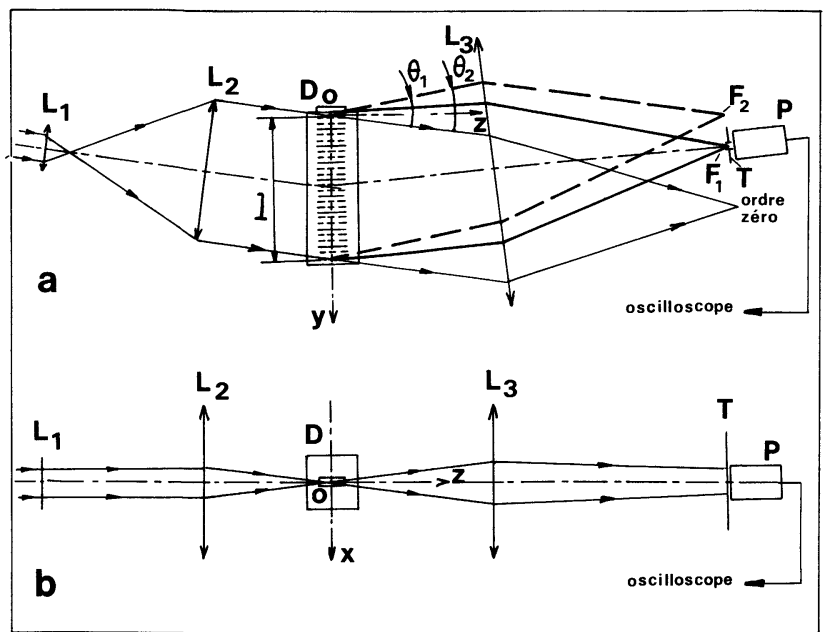

Fig. 1. - Schéma de principe du montage spectroscopique a) Dans le plan $O Y Z$ d'incidence et de déviation. b) Dans le plan $O X Z$ perpendiculaire au plan de déviation. $\mathrm{L}_{1}$ : lentille cylindrique ; $L_{2}$ et $L_{3}$ : lentilles sphériques; $D$ : déviateur; $T$ : fente d'analyse; $\mathrm{P}$ : photodétecteur. Pour une lumière incidente monochromatique, on a représenté le faisceau direct (-) et les faisceaux diffractés par une onde acoustique de fréquence $F_{1}$ $(\longrightarrow)$ et $F_{2}>F_{1}(--)$.

[Experimental set up : a) $O Y Z$ incidence and deflecting plane. b) $O X Z$ plane perpendicular to $O Y Z . \mathrm{L}_{1}$ : cylindric lens; $\mathrm{L}_{2}$ and $\mathrm{L}_{3}$ : spheric lenses; $\mathrm{D}:$ Bragg cell ; $\mathrm{T}$ : analysis slit ; $\mathrm{P}:$ photodetector. For a monochromatic incident light, the diffracted light beams by an acoustic wave of $F_{1}$ and $F_{2}>F_{1}$ frequencies are indicated by $(-)$ and $(--)$ respectively and by $(-)$ for the direct light beam.]

2. Montage expérimental. - L'ensemble placé avant la cellule d'interaction acousto-optique est destiné à donner au faisceau optique une section rectangulaire de $28 \mathrm{~mm}$ de largeur et de quelques dizaines de micromètres d'épaisseur à l'endroit où s'effectue l'interaction. La lentille placée après le déviateur effectue la transformée de Fourier des ondes lumineuses diffractées qui se présentent dans le plan focal sous forme de segments lumineux dont la plus grande dimension est perpendiculaire au plan de déviation. Le dispositif d'analyse est constitué d'une fente fixe parallèle aux figures de diffraction, et dont les dimensions permettent une analyse correcte des signaux. Un photomultiplicateur placé derrière la fente, détecte les signaux qui sont visualisés sur l'écran d'un oscilloscope comme en témoignent les photographies des figures 2 et 3.

3. Caractéristiques intrinsèques du montage spectroscopique. - 3.1 POUVOIR SÉPARATEUR, RÉSOLVANCE. - On suppose que le faisceau acoustique interagit avec une onde lumineuse plane et uniforme. La divergence naturelle du faisceau lumineux diffracté est alors donnée par l'expression (critère de Rayleigh) :

$$
\mathrm{d} \theta=\frac{\lambda}{l}
$$

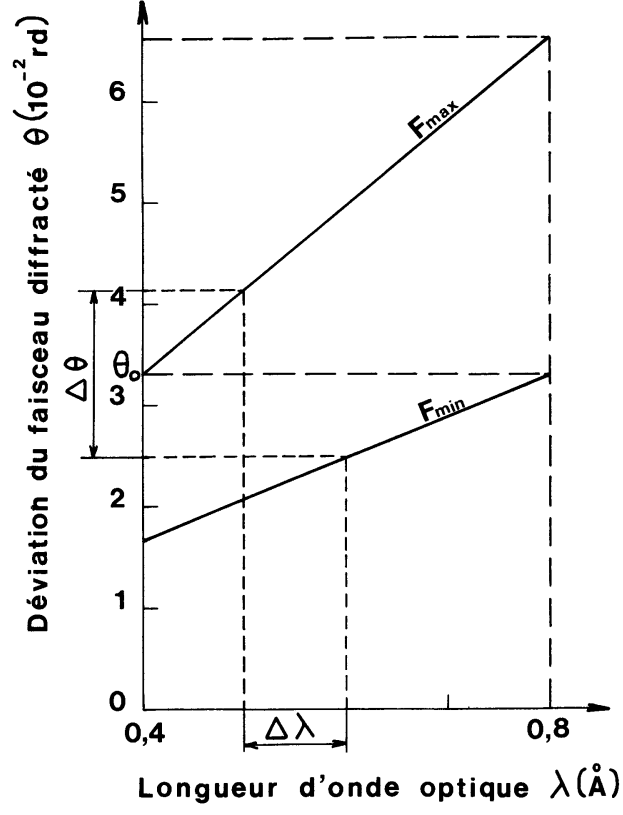

Fig. 2. - Déviation angulaire du faisceau lumineux diffracté en fonction de la longueur d'onde optique. Avec $F_{\min }=150 \mathrm{MHz}$; $F_{\max }=300 \mathrm{MHz}$ et $V=3630 \mathrm{~m} / \mathrm{s}$.

[Angular deviation of the diffracted light beam versus optical wavelength. With $F_{\min }=150 \mathrm{MHz} ; \quad F_{\max }=300 \mathrm{MHz}$ and $V=3630 \mathrm{~m} / \mathrm{s}$.]

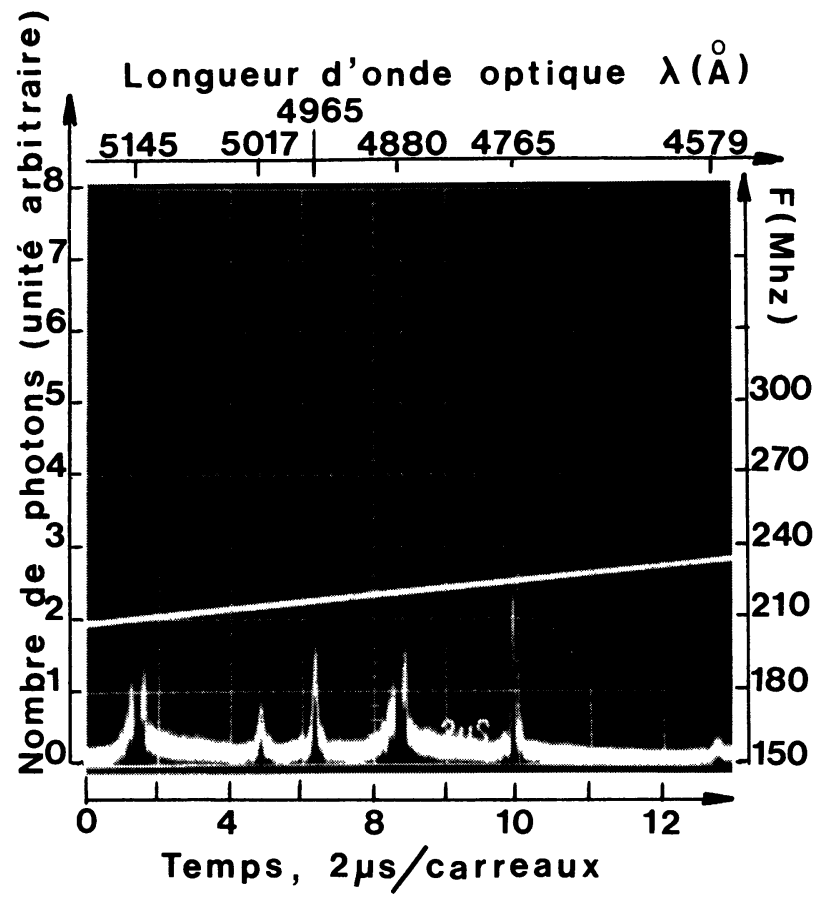

Fig. 3. - Spectre du laser à argon analysé par balayage temporel. L'échelle supérieure permet l'identification des différentes raies du laser. La droite inclinée représente la variation de la fréquence acoustique en fonction du temps.

[Ar ion laser spectrum observed by scanning. The upper scale gives the wavelengths of the lines. The acoustic frequencies versus time are indicated by the inclined straight line.] 
dans laquelle $l$ représente la longueur d'interaction. Ainsi d'après (1) et (2), nous pouvons définir le pouvoir séparateur intrinsèque

$$
(\mathrm{d} \lambda)_{0}=\frac{V \lambda}{F l} \cos \theta
$$

et la résolvance intrinsèque :

$$
R_{0}=\frac{\lambda}{(\mathrm{d} \lambda)_{0}}=\frac{F l}{V \cos \theta} .
$$

Dans notre expérience, $l=28 \mathrm{~mm}$ et $V=3630 \mathrm{~m} / \mathrm{s}$; ainsi pour $\lambda=5145 \AA$ et $F=300 \mathrm{MHz}$, nous obtenons $(\mathrm{d} \lambda)_{0}=2,2 \AA$ et $R_{0}=2314$.

Des relations précédentes, nous pouvons dès à présent remarquer l'indépendance de la résolution vis-à-vis de la vitesse de balayage des fréquences acoustiques.

Cette particularité résultant du principe même, constitue l'avantage majeur du dispositif.

Par contre, la résolution n'est pas uniforme au cours du balayage et les courtes longueurs d'onde sont les mieux résolues (Fig. 5 courbe $a$ ). Il est préférable de travailler avec des fréquences acoustiques élevées et avec une longueur d'interaction grande dans la mesure où le facteur d'absorption des ultrasons dans le cristal le permet. En pratique, on adopte une position optimum de la fente d'analyse dans le plan focal de la lentille de sortie, afin de travailler avec des fréquences acoustiques les plus élevées de l'intervalle d'accord $\Delta F$ et fixant ainsi $\sin \theta$.

Toutefois, la position de la fente est tributaire du domaine spectral à analyser que nous allons maintenant préciser.

3.2 Domaine spectral analysé. - Pour un déviateur donné, dont $F_{\min }$ et $F_{\max }$ (limites inférieure et supérieure de $\Delta F$ ) sont fixées, le domaine spectral analysé $\Delta \lambda$ est directement déterminé par la position de la fente, définie par $\theta_{0}$ (Fig. 2).

D'après (1), $\Delta \lambda$ s'écrit :

$$
\Delta \lambda=\lambda_{\min } \frac{\Delta F}{F_{\min }}
$$

avec

$$
\lambda_{\min }=\frac{V}{F_{\max }} \sin \theta_{0}, \text { origine de } \Delta \lambda .
$$

Pour le dispositif proposé, $\Delta F=150 \mathrm{MHz}$ et $F_{\min }=150 \mathrm{MHz}$, ainsi le domaine spectral analysé a pour expression :

$$
\Delta \lambda=\lambda_{\min }=\frac{\lambda_{\max }}{2},
$$

$\lambda_{\max }$ étant la longueur d'onde maximale du domaine spectral analysé. Le dispositif couvre une largeur spectrale de 0,4 à $0,8 \mu \mathrm{m}$, soit largement le spectre visible.
Dans le cas où le domaine spectral à analyser est plus étroit que celui exprimé en (5), la figure 2 montre que $\theta_{0}$ peut prendre plusieurs valeurs de l'intervalle $\Delta \theta$. En pratique, on adopte la plus grande valeur possible de cet intervalle afin de travailler avec des fréquences acoustiques élevées et par conséquent d'optimiser la résolution.

4. Résultats expérimentaux. - 4.1 PouvoIR SÉPARATEUR. - Les résultats expérimentaux sont établis à partir des enregistrements photographiques représentés sur les figures 3 et 4 . A la fonction

$$
F=\frac{\Delta F}{t_{\mathrm{b}}} t+F_{\min },
$$

qui exprime la variation des fréquences acoustiques au cours du balayage, se superposent les différentes figures de diffraction des composantes spectrales de la lumière incidente (Fig. 3) ; ce qui permet d'étalonner le dispositif spectroscopique.

A partir des relations (1) et (6), nous pouvons établir le pouvoir séparateur expérimental, soit :

$$
|\mathrm{d} \lambda|=\frac{\lambda V}{F l} \frac{N}{N^{\prime}},
$$

expression dans laquelle :

$$
N=\frac{l}{V} \Delta F,
$$

représente le nombre théorique de directions distinctes qui caractérise un déviateur, et.

$$
N^{\prime}=\frac{t_{\mathrm{b}}}{\mathrm{d} t},
$$

le nombre expérimental de directions distinctes; $t_{\mathrm{b}}$ représentant le temps de balayage de l'intervalle $\Delta F$; et $\mathrm{d} t$ le temps mis pour analyser la tache de diffraction considérée, et qui correspond à une largeur $\mathrm{d} x$ définie d'après le critère de Rayleigh (Fig. 4).

En posant $N / N^{\prime}=\alpha$, le pouvoir séparateur expérimental s'écrit :

$$
\mathrm{d} \lambda=(\mathrm{d} \lambda)_{0} \alpha,
$$

$\alpha$ est un facteur plus grand que 1 qui caractérise le dispositif expérimental et qui, d'après les courbes de la figure 5 vaut environ 1,5.

Les facteurs déterminant $\alpha$ sont de natures diverses :

D'une part, nous avons supposé que l'amplitude de l'onde lumineuse dans la direction de l'interaction était plane et uniforme. Ceci n'est pas rigoureuse-, ment exact et cette hypothèse optimise la valeur de $(\mathrm{d} \lambda)_{0}$.

D'autre part, les taches de diffraction se trouvent élargies et déformées par la présence d'aberrations géométriques. 

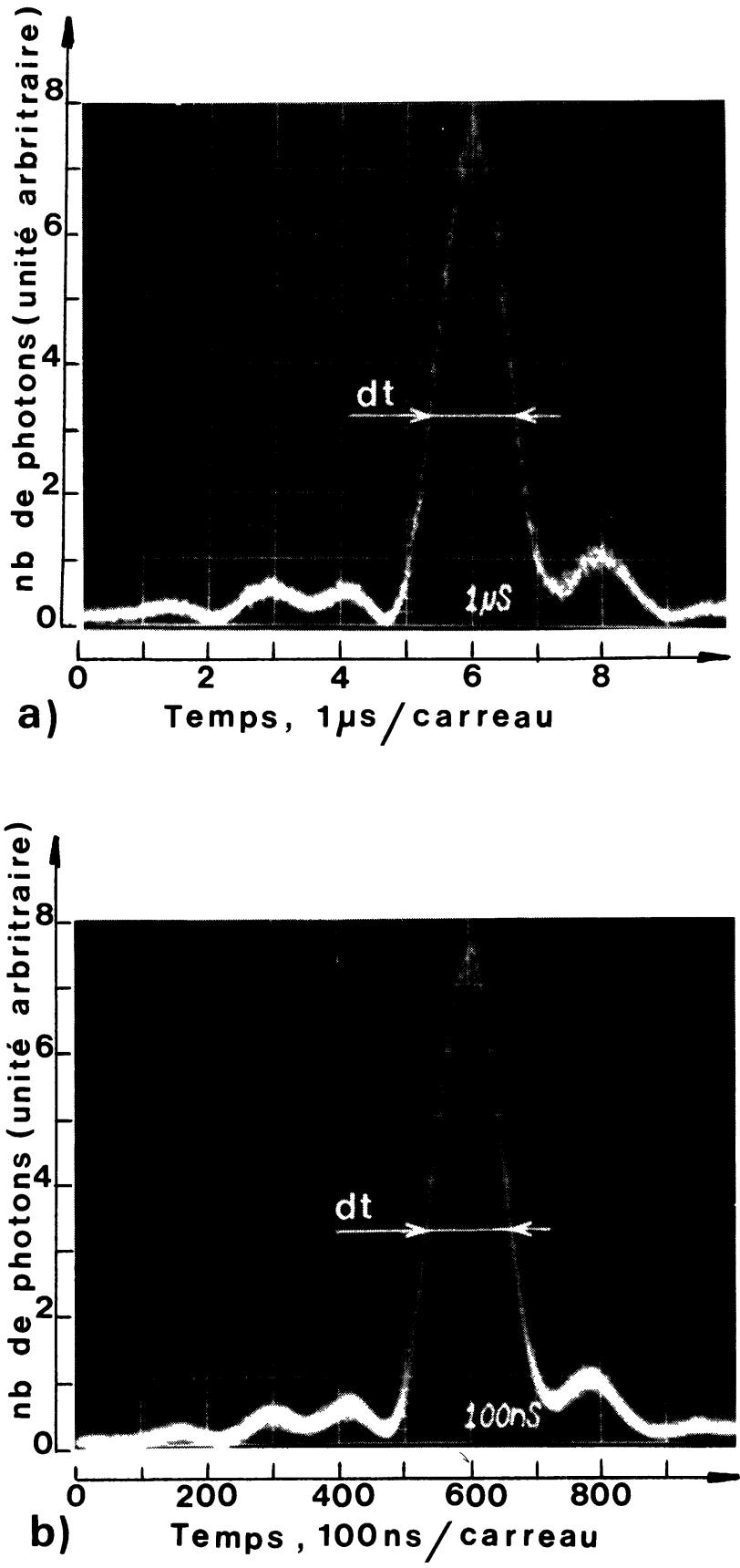

Fig. 4. - Figures de diffraction pour $\lambda=5145 \AA$ et $F=250 \mathrm{MHz}$ a) La vitesse de balayage des fréquences acoustiques vaut $\Delta F / t_{\mathrm{b}}=0,15 \mathrm{MHz} / \mu$ s et le pouvoir de résolution

$$
\mathrm{d} \lambda=4,1 \AA \pm 0,1 \AA
$$

b) $\Delta F / t_{\mathrm{b}}=1,5 \mathrm{MHz} / \mu \mathrm{s} ; \mathrm{d} \lambda=4,0 \AA \pm 0,1 \AA$.

[Diffraction patterns for $\lambda=5145 \AA$ and $F=250 \mathrm{MHz}$. a) The scanning rate of acoustic frequencies is $\Delta F / t_{\mathrm{b}}=0.15 \mathrm{MHz} / \mu \mathrm{s}$ and the resolving power is $\mathrm{d} \lambda=4.1 \AA \pm 0.1 \AA ; b) \Delta F / t_{\mathrm{b}}=1.5 \mathrm{MHz} / \mu \mathrm{s}$; $\mathrm{d} \lambda=4.0 \AA \pm 0.1 \AA$.

Une analyse correcte et simultanée de toutes les composantes spectrales de la source est rendue difficile par la présence d'aberrations chromatiques, dues aux éléments réfractants qui composent le montage optique.

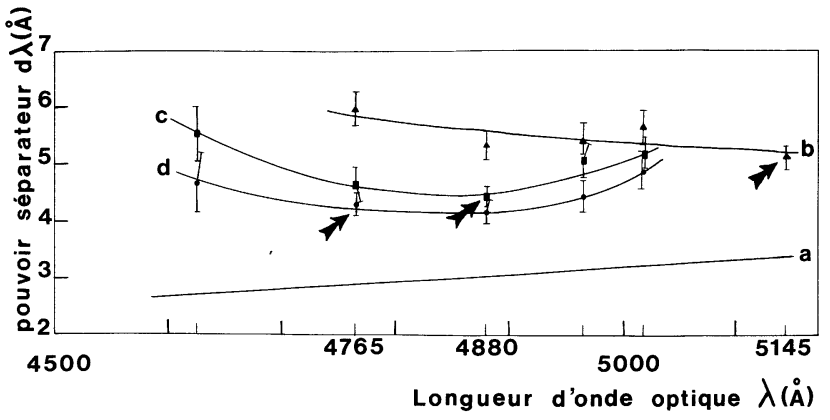

Fig. 5. - Pouvoir séparateur en fonction de la longueur d'onde optique, pour $\theta=0,029 \mathrm{rad}$. (a) Courbe théorique. $(b),(c),(d)$ Exemples de courbes expérimentales. Pour chacune d'elles, la flèche identifie la longueur d'onde pour laquelle la position de la fente est optimum.

[Resolving power versus optical wavelength, for $\theta=0.029 \mathrm{rad}$. $(a)$ Theoretical curve. $(b),(c),(d)$ Examples of experimental curves. On each curve, the arrow indicates the optical wavelength for which the position of the analysis slit is optimum.]

En pratique la position de la fente d'analyse est optimisée pour une seule longueur d'onde. Pour les autres longueurs d'onde le pouvoir séparateur se trouve affecté comme le montrent les courbes $b$, $c$ et $d$ de la figure 5 .

Nous vérifions expérimentalement une meilleure résolution pour les courtes longueurs d'onde (points repérés par les flèches, Fig. 5).

Le dispositif présente un pouvoir séparateur d'environ $4,5 \AA$ pour les principales raies du laser à argon, soit dans un domaine spectral d'environ $500 \AA$.

4.2 Influence du TEMPS De Balayage. - Comme on peut le constater sur la figure 4 , la résolution du dispositif spectroscopique est indépendante de la vitesse de balayage.

En pratique, nous sommes limités par le temps de réponse du circuit électronique de mesure branché après le photodétecteur. Dans le cas de notre expérience où le circuit de détection n'est pas particulièrement performant, nous balayons tout le spectre visible en $100 \mu$ s et analysons les raies du laser à argon en $16 \mu \mathrm{s}$ environ (Fig. 3).

Toutefois si le temps de balayage $t_{\mathrm{b}}$ n'est pas négligeable devant le temps de transit $\tau$ d'un phonon dans le cristal, la présence simultanée de plusieurs fréquences acoustiques à l'intérieur du cristal a deux conséquences pratiques :

La première se traduit pour le faisceau diffracté par un effet de lentille cylindrique de focale :

$$
F_{\mathrm{c}}= \pm \frac{V^{2} t_{\mathrm{b}}}{\lambda \Delta F}, \text { [7] }
$$

On s'affranchit de cet inconvénient par une simple translation de la fente d'analyse dans la direction $O Z$ (Fig. 1). $F_{\mathrm{c}}$ étant inversement proportionnelle à $\lambda$; 
dans le cas où $F_{\mathrm{c}}$ est positif, on peut même avoir compensation partielle des aberrations chromatiques du montage.

La deuxième conséquence affecte la largeur spectrale analysable. En effet la largeur $\Delta F^{\prime}$ des fréquences acoustiques qui contribue réellement à l'interaction est inférieure à $\Delta F$ et vaut :

$$
\Delta F^{\prime}=\Delta F \frac{t_{\mathrm{b}}-\tau}{t_{\mathrm{b}}} .
$$

D'après (5), la largeur spectrale analysée devient :

$$
\Delta \lambda^{\prime}=\Delta \lambda \frac{t_{\mathrm{b}}-\tau}{t_{\mathrm{b}}}
$$

Pour un cristal de $\mathrm{PbMoO}_{4}$, de longueur $l=28 \mathrm{~mm}$ $(\tau=8 \mu \mathrm{s})$, un temps de balayage $t_{\mathrm{b}}=2 \tau=16 \mu \mathrm{s}$ réduit de moitié le domaine spectral analysé.

5. Conclusion. - Nous avons déterminé les caractéristiques expérimentales d'un dispositif d'analyse spectrale éclairé par une source laser. Cependant, il n'est pas impensable d'envisager la réalisation d'un spectromètre ultra-rapide pour l'analyse des sources spatialement incohérentes.

Les performances du dispositif pourraient être améliorées en utilisant pour le déviateur d'autres substances telles que l'acide alpha-iodique $\left(\alpha-\mathrm{HIO}_{3}\right)$ dont les caractéristiques acousto-optiques sont intéressantes [8]. Toutefois, ses propriétés hygroscopiques rendent difficile l'élaboration de la cellule de déviation (polissage des faces) et engendrent une détérioration rapide du matériau qui limite son utilisation dans le temps.

\section{Bibliographie}

[1] Warner, A. W., White, D. L. and Bonner, W. A., J. Appl. Phys. 43 (1972) 4489.

[2] Bauza, J. M., Carles, C. et Torguet, R., I. Théorie. Acustica 30 (1974) 137.

[3] Bauza, J. M., Carles, C. et Torguet, R., II. Expériences. Acustica 31 (1974) 161.

[4] Dieulesaint, E. et Royer, D., Ondes élastiques dans les solides (Ed. Masson \& Cie, Paris) 1974, p. 322.
[5] Watanabe, A. and Yano, T., Proceedings of the 6th Conference on Solid State Devices, Tokio, 1974. Supplement to the journal of the Japan Society of Applied Physics, Vol. 44 (1975).

[6] Yano, T. and Watanabe, A., Appl. Opt. 15 (1976).

[7] Leroy, Dickson, D., Appl. Opt. 11 (1972).

[8] Pinnow, D. A. and Dixon, R. W., Appl. Phys. Lett. 13 (1968). 